\title{
Effect of BMI on the Thermogenic Response to Cold Exposure and Associated Changes in Metabolism and Browning Markers in Adult Humans
}

\author{
Laura Aline Mengel ${ }^{a, b, c} \quad$ Bahareh Nemati Moud $^{c} \quad$ Hatti Seidla $^{a}$ \\ Alberto Mesas-Fernández ${ }^{c} \quad$ Claudine Seeliger $^{c}$ Beate Brandl ${ }^{b, c}$ \\ Thomas Skurk $^{b, c}$ Christina Holzapfel ${ }^{a}$ Melina Claussnitzer ${ }^{\text {d, e }}$ Hans Hauner $^{\text {a, b, c }}$ \\ anstitute of Nutritional Medicine, School of Medicine, Technical University of Munich, Munich, Germany; \\ bZIEL Institute for Food and Health, Technical University of Munich, Freising-Weihenstephan, Germany; \\ 'Else Kröner-Fresenius Center for Nutritional Medicine, School of Life Sciences, Technical University of Munich, \\ Freising-Weihenstephan, Germany; ${ }^{\mathrm{d}}$ Metabolism Program, Broad Institute of MIT and Harvard, Cambridge, MA, USA; \\ eBeth Israel Deaconess Medical Center, Harvard Medical School, Boston, MA, USA
}

\section{Keywords}

Brown adipose tissue $\cdot$ Cold exposure $\cdot$ Nonshivering thermogenesis · Resting energy expenditure $\cdot$ Browning markers

\begin{abstract}
Introduction: Brown adipose tissue (BAT) serves to produce heat by nonshivering thermogenesis. Activation of BAT increases energy expenditure and is seen as a putative strategy to treat obesity. There are conflicting data on the capacity for cold-induced thermogenesis in individuals with higher BMI. Methods: To investigate the effect of BMI on cold-induced stimulation of energy expenditure, changes in the metabolic profile, and the expression of browning markers in subcutaneous white adipose tissue (scWAT), healthy adults ( $N=173,50.9 \%$ females) with a median age of 26.0 (interquartile range [IQR]: 23.0; 28.0) years and a median body mass index (BMI) of 23.6 [IQR: $21.9 ; 26.6] \mathrm{kg} / \mathrm{m}^{2}$ were exposed to short-term mild cold exposure (CE). Resting energy expenditure (REE) was measured by indirect calorimetry and blood sampling was conducted at baseline and after CE. In a subgroup of participants with obesity, subcutaneous
\end{abstract}

Karger@karger.com www.karger.com/ofa

Karger"

BOPEN ACCESS
(C) 2022 The Author(s)

Published by S. Karger AG, Basel

This is an Open Access article licensed under the Creative Commons Attribution-NonCommercial-4.0 International License (CC BY-NC) (http://www.karger.com/Services/OpenAccessLicense), applicable to the online version of the article only. Usage and distribution for commercial purposes requires written permission. abdominal fat biopsies were taken before and after CE. Results: The cold-induced median increase in REE was 74 (IQR: $-28 ; 241) \mathrm{kcal} /$ day $(p<0.001)$. This increase negatively correlated with BMI $(p<0.001)$. Participants with BMI 18.5-24.9 $\mathrm{kg} / \mathrm{m}^{2}$ displayed a significant median increase of $103 \mathrm{kcal} /$ day $(p<0.001)$, participants with overweight or obesity were not able to increase REE $(23, p=0.468$ or $-30 \mathrm{kcal} /$ day, $p=$ 0.917 , respectively). In participants with obesity, expression of cell death activator in scWAT after CE was upregulated in females ( $p=0.034)$. Conclusions: Persons with overweight and obesity do not increase REE in response to $C E$, presumably reflecting lower BAT activity. Likewise, the metabolic response to cold is diminished in participants with elevated BMI.

(c) 2022 The Author(s).

Published by S. Karger AG, Basel

\section{Introduction}

The existence of brown adipose tissue (BAT) in humans was first described in the early 1900s in dissection studies $[1,2]$. In contrast to rodents, the evidence is unclear in humans if BAT plays a role in the development of
Correspondence to:

Laura Aline Mengel, laura.mengel@tum.de

Hans Hauner, hans.hauner@tum.de 
obesity and its metabolic disturbances. Early attempts to develop sympathomimetic compounds that activate BAT turned out to either be ineffective to treat obesity or to cause adverse effects on the cardiovascular system [3]. Interest in BAT was renewed more than 10 years ago with studies using 18-fluoro-labeled 2-deoxy-glucose positron emission tomography combined with computed tomography $\left({ }^{18}\right.$ F-FDG-PET-CT) imaging [4-6]. These studies showed the presence and activation of BAT in adult humans particularly after cold exposure (CE). The presence of active BAT was lower in elderly persons as well as in people with higher body mass index (BMI) $[7,8]$. In contrast to white adipose tissue (WAT), BAT does not serve for lipid storage, but facilitates nonshivering thermogenesis (NST) by burning lipids, thus releasing energy as heat. A unique feature of brown adipocytes is the expression of uncoupling protein 1 (UCP1) which is present in the membrane of the highly abundant mitochondria in these cells [9].

In studies using ${ }^{18} \mathrm{~F}$-FDG-PET-CT analysis, the gold standard for detecting active BAT, a negative correlation between BAT mass and BMI, was reported $[5,7,10]$. In other studies, BAT mass was found to be reduced or not detectable in participants with obesity $[11,12]$. Conventional MRI analyses showed a positive correlation of BMI with the fat fraction of the supraclavicular area, a location where BAT is mostly found, suggesting that this depot contains less BAT in individuals with higher BMI [13]. Activation of BAT after CE using ${ }^{18}$ F-FDG-PET-CT analysis was found to be associated with improved wholebody glucose homeostasis and insulin sensitivity in humans $[14,15]$. Upon acute CE in lean, young adults, BAT was shown to be present and activated [16]. It was also reported that in subjects with a BMI of $28.1-36.8 \mathrm{~kg} / \mathrm{m}^{2}$, a substantial amount of BAT can be recruited after cold acclimation [17]. Large-scale retrospective studies suggested that individuals with active BAT have fewer cardiovascular symptoms and generally better blood lipid and blood glucose levels [18, 19].

Despite the growing number of studies, most were small in sample size (mostly between 10 and 30 participants), and there is still some inconsistency in the literature. In the present study, we were interested to assess changes in energy expenditure and metabolic parameters induced by an individualized CE in a large sample of 173 healthy adults across a wide BMI range. We analyzed whether the cold-induced change in resting energy expenditure (REE) is associated with BMI and changes in metabolic parameters. Furthermore, we took biopsies of subcutaneous WAT (scWAT) from 17 participants with obesity before and after CE to examine changes in messenger RNA expression of selected browning marker genes.

\section{Material and Methods}

Study Design

This intervention study was conducted at two study sites of the Technical University of Munich (Munich and Freising) using identical equipment and with the same personnel. The study protocol was reviewed and approved by the Ethics Commission of the School of Medicine of the Technical University of Munich (\#236/16S). Recruitment started in October 2016 and ended in April 2019. Participants were recruited by advertising via bulletins, university mailing lists, and a newspaper article. Each volunteer gave their written informed consent. The study was registered at the German Clinical Trials Register DRKS (accession number: DRKS00010489). A subgroup of this cohort was already described previously [20].

Individuals were enrolled for the study when they met predefined eligibility criteria as described recently in detail [20]. Briefly, participants were 18-60 years old, nonsmoking, and without severe diseases. The presence of diabetes was another exclusion criterion. In case of acute infection, such as common cold, appointments were postponed. Attrition of participants is displayed in the online supplementary Figure S1 (for all online suppl. material, see www.karger.com/doi/10.1159/000522218).

\section{Anthropometric Measurements}

Measurements started in the morning between 07:30 a.m. and 08:00 a.m. Participants were asked to refrain from caffeine and exercise for at least $24 \mathrm{~h}$ and from caloric beverages or food for at least $10 \mathrm{~h}$. Body height $(\mathrm{cm})$ was measured to the nearest $0.1 \mathrm{~cm}$ using a stadiometer (Seca, Hamburg, Germany). Body composition and weight were measured using the TANITA Body Composition Analyzer Type BC-418 MA (Tanita Europe GmbH, Sindelfingen, Germany). Systolic and diastolic blood pressure and heart rate were assessed in triplicate in a sitting position before each blood sampling using Omron M8 comfort (Omron Medizintechnik Handelsgesellschaft mbH, Mannheim, Germany).

\section{Measurement of Temperatures}

For assessing skin temperature, participants were equipped with eight wireless thermosensors (iButtons DS-1921 H, Thermochron; Maxim, Dallas, TX, USA) at positions of the body defined by ISO9886 [21]. One additional sensor was attached to the supraclavicular fossae. Outside temperature data were obtained from the website of the German Meteorological Service (Deutscher Wetterdienst [DWD], www.dwd.de) for the study site in Munich. For Freising, data were obtained from the meteorological station in Freising (https://www.wetter-weihenstephan.de/) which is run by DWD and LfL (Bayerische Landesanstalt für Landtechnik). Room temperature was kept stable and was monitored during the experiment.

\section{Measurement of REE}

REE was assessed at two time points: at thermoneutrality (TN) and after 2-h CE, for 30 min each. This was done by indirect calo- 
rimetry using a ventilated canopy hood system (COSMED Quark RMR, Fridolfing, Germany). On each morning before the participant arrived, a volume calibration was done, and before each indirect calorimetry measurement, a gas calibration of the system was conducted. By applying the shortened Weir equation, conversion of the measurements of $\mathrm{VO}_{2}$ (consumption of oxygen $[\mathrm{mL} / \mathrm{min}]$ ) and $\mathrm{VCO}_{2}$ (production of $\mathrm{CO}_{2}[\mathrm{~mL} / \mathrm{min}]$ ) to $\mathrm{REE}$ (kcal/day) was assessed [22]. The respiratory exchange ratio was calculated by dividing $\mathrm{VCO} 2$ by $\mathrm{VO} 2$. To ensure data quality, REE measurements had to achieve at least 4 continuous minutes in which the variances of $\mathrm{VCO}_{2}$ and $\mathrm{VO}_{2}$ were less than $10 \%$ each [23]. This steadystate was not met by 10 participants and they were excluded from REE analysis.

\section{Individualized Cooling Protocol}

After anthropometric and cardiovascular measurements, participants were bedded between two water perfused blankets (MaxiTherm Lite Blankets; Cincinnati Sub-Zero, Cincinnati, OH, USA) that were connected to a cooling device (WiseCircu type WCR-P8; Witeg Labortechnik, Wertheim, Germany). At TN, the water inlet temperature was set to $32^{\circ} \mathrm{C}$, or lower if this temperature was sensed as too warm. After the first indirect calorimetry and blood sampling, the cooling protocol started. The water temperature was decreased stepwise, as described earlier [20]. As soon as participants reported shivering or showed any signs thereof, the shivering temperature was noted and the water inlet temperature was increased by $2^{\circ} \mathrm{C}$. Then, CE was maintained for $120 \mathrm{~min}$ at this defined temperature (Texp). If shivering occurred during that time, the temperature was increased further until shivering stopped. Participants with shivering during the second REE measurement $(n=2)$ were excluded from the analysis.

\section{Blood Analyses}

Each indirect calorimetry was followed by blood sampling. Fasting plasma glucose was determined using a point-of-care glucometer system (HemoCue Glucose 201+ System; Radiometer $\mathrm{GmbH}$, Willich, Germany). Enzyme-linked immunosorbent assays were used to measure plasma leptin, adiponectin (Duoset ELISA human; R\&D, Wiesbaden, Germany), and insulin (DRG Insulin ELISA; DRG Instruments, Marburg, Germany). Plasma nonesterified fatty acids (NEFAs) were assessed by using an enzymatic colorimetric method (Wako Chemicals, Neuss, Germany). Serum triglycerides (TGs), C-reactive protein (CRP), and free tri-iodothyronine were measured using established commercial test kits by a certified laboratory service (SynLab Labordienstleistungen, Munich, Germany). One participant was excluded from analysis as her thyroid levels were out of the accepted range and for two participants blood sampling after CE was not successful for technical reasons. For one further participant, CE values for TG, $\mathrm{CRP}$, and free triiodothyronine were not available. Insulin sensitivity was calculated by homeostasis model assessment (HOMAIR): HOMA-IR = plasma glucose $(\mathrm{mmol} / \mathrm{L}) \times$ plasma insulin $(\mu \mathrm{U} /$ $\mathrm{mL}) / 22.5$ [24].

\section{Adipose Tissue Biopsy and Analysis}

To obtain scWAT samples in a subgroup with overweight or obesity, needle aspiration biopsy at the region lateral from the umbilicus was conducted after each indirect calorimetry, subsequent to blood sampling, under sterile conditions providing enough scWAT ( $>1 \mathrm{~g}$ wet tissue per biopsy). This was done on a voluntary basis and with written informed consent. For RNA processing, the fat tissue obtained was aliquoted into tubes containing sterilized zirconia glass beads (Carl Roth, Karlsruhe, Germany), RLT buffer (RNeasy Mini Kit; Qiagen, Hilden, Germany), and 1\% (vol/vol) $\beta$-mercaptoethanol (Sigma-Aldrich, St. Louis, MO, USA).

Messenger RNA (mRNA) was isolated according to the protocol of the mirVana miRNA Isolation Kit (Thermo Fisher Scientific, Dreieich, Germany) for fat tissue. This was followed by transcribing complementary DNA according to the protocol of HighCapacity cDNA Reverse Transcription Kits (Thermo Fisher Scientific). Quantitative PCR was carried out with SYBRgreen MasterMix (QIAGEN GmbH, Düsseldorf, Germany) to measure the expression of beiging markers along with PPIA, GAPDH, and IPO8 as housekeeping genes. We performed gene expression analyses in scWAT samples of 17 individuals (12 females) before and after $\mathrm{CE}$. The sequences of the primers used are listed in online supplementary Table S1.

\section{Statistical Analysis}

Data were analyzed using GraphPad Prism Version 5.2 (GraphPad Software, Inc., San Diego, CA, USA) and RStudio (RStudio Inc., Boston, MA, USA). The normality of data was formally tested by visual inspection of histograms. As most parameters were not normally distributed, we used nonparametric tests. Baseline characteristics and outcome variables, including REE, metabolic parameters, and skin temperatures, were compared between $\mathrm{TN}$ and CE using Wilcoxon signed-rank test and are presented as median including interquartile range [IQR]. For comparing the different BMI categories, Kruskal-Wallis analysis of variance was applied. For assessing differences between two groups, and between sexes, the Mann-Whitney U test was applied. Pearson and Spearman's rank correlation was used to identify correlations between variables. In simple linear regressions, Texp, sex, BMI, fat mass (FM), fat-free mass (FFM), age, study site, and ambient temperature were used as independent variables with $\triangle \mathrm{REE}$ and $\triangle \mathrm{REE}(\%)$ as dependent variables. Additionally, two multiple linear regression approaches were performed using BMI, ambient temperature, Texp, and FFM or FM, respectively, as the independent variables. A sensitivity analysis was done which excluded outliers that differed more than 3 SDs from the mean. Relative gene expression was calculated by the $\Delta \Delta \mathrm{CT}$ method and normalized to the mean expression of the housekeeper genes. Bonferroni correction was applied. Results were considered statistically significant at $p<0.05$. Due to the explorative nature of the study, no power calculation was done.

\section{Results}

Baseline characteristics of the study group are presented in Table 1. The median age of the participants was 26.0 [23.0-28.0] years, and the median BMI was 23.6 [21.9$26.6] \mathrm{kg} / \mathrm{m}^{2}$. The normal weight group (NW, BMI 18.5$24.9 \mathrm{~kg} / \mathrm{m}^{2}$ ) was significantly younger (25.0 [23.0-27.0] years) compared to the group with overweight (OW, BMI $25-29.9 \mathrm{~kg} / \mathrm{m}^{2}, 27.0[25.0-28.5]$ years; $\left.p=0.012\right)$ and the group with obesity $\left(\mathrm{OB}, \mathrm{BMI} \geq 30 \mathrm{~kg} / \mathrm{m}^{2}, 28.0\right.$ [26.0-31.8] years; $p<0.001$ ) (Table 2). 
Table 1. Participant characteristics

\begin{tabular}{llllll}
\hline & All $(n=170)$ & NW $(n=117)$ & OW $(n=27)$ & OB $(n=26)$ & $p$ value \\
\hline Sex $(\mathrm{M} / \mathrm{F})$ & $83 / 87$ & $58 / 59$ & $14 / 13$ & $11 / 15$ \\
Age, years & $26.0[23.0 ; 28.0]$ & $25.0[23.0 ; 27.0]$ & $27.0[25.0 ; 28.5]$ & $28.0[26.0 ; 31.8]$ \\
Weight, kg & $74.8[65.1 ; 86.2]$ & $67.9[61.1 ; 77.4]$ & $84.8[77.3 ; 88.8]$ & $101.1[96.5 ; 110.7]$ & $<0.001$ \\
Height, $\mathrm{cm}$ & $175.5[169.5 ; 183.2]$ & $176.0[170.5 ; 185.1]$ & $176.5[167.8 ; 183.8]$ & $174.3[168.0 ; 177.7]$ & 0.487 \\
BMl, kg/m & $23.6[21.9 ; 26.6]$ & $22.6[21.3 ; 23.7]$ & $26.8[26.2 ; 27.8]$ & $34.1[31.7 ; 36.3]$ & $<0.001$ \\
FM, $n(\%)$ & $23.1[14.4 ; 29.8]$ & $19.0[12.5 ; 25.2]$ & $26.2[19.1 ; 35.1]$ & $38.2[27.7 ; 43.1]$ & $<\mathbf{0 . 0 0 1}$ \\
FFM, kg & $57.8[47.4 ; 68.6]$ & $53.2[45.8 ; 81.5]$ & $64.8[51.4 ; 69.6]$ & $63.7[54.6 ; 77.2]$ & $\mathbf{0 . 0 0 1}$ \\
\hline
\end{tabular}

Values are presented as median with [IQR]; differences between BMI groups were assessed by Kruskal-Wallis analysis with Bonferroni correction. BMI, body mass index; F, females; FM, fat mass; FFM, fat-free mass; IQR, interquartile range; M, males; NW, normal weight (BMI $\left.18.5-24.9 \mathrm{~kg} / \mathrm{m}^{2}\right) ; O B$, obese (BMI $\geq 30 \mathrm{~kg} / \mathrm{m}^{2}$ ); OW, overweight (BMI $25.0-29.9 \mathrm{~kg} / \mathrm{m}^{2}$ ).

Table 2. Multiple linear regression of REE TN, $\triangle \mathrm{REE}$, and $\triangle \mathrm{REE}(\%)$ as dependent variables

\begin{tabular}{|c|c|c|c|c|c|c|c|c|}
\hline & \multicolumn{4}{|c|}{ REE TN $\sim \mathrm{BMI}+$ sex + age + FFM } & & & & \\
\hline & estimate & std error & $t$ value & $p$ value & & & & \\
\hline Sex (male) & -126.89 & 59.01 & -2.15 & 0.033 & & & & \\
\hline Age, years & -5.32 & 2.60 & -2.05 & 0.042 & & & & \\
\hline \multirow[t]{2}{*}{ FFM } & 22.98 & 2.48 & 9.26 & $<0.001$ & & & & \\
\hline & estimate & std error & $t$ value & $p$ value & estimate & std error & $t$ value & $p$ value \\
\hline Texp & -35.7 & 7.11 & -5.02 & $<0.001$ & -2.18 & 0.43 & -5.05 & $<0.001$ \\
\hline $\mathrm{BMI}, \mathrm{kg} / \mathrm{m}^{2}$ & -12.07 & 3.83 & -3.15 & 0.002 & -0.73 & 0.23 & -3.13 & 0.002 \\
\hline FFM & -3.38 & 1.56 & -2.17 & 0.032 & -0.29 & 0.09 & -3.08 & 0.002 \\
\hline Tamb & estimate & std error & $t$ value & $p$ value & estimate & std error & $t$ value & $p$ value \\
\hline Texp & -36.89 & 7.08 & -5.21 & $<0.001$ & -2.19 & 0.43 & -5.08 & $<0.001$ \\
\hline BMI, $\mathrm{kg} / \mathrm{m}^{2}$ & -31.97 & 7.6 & -4.21 & $<0.001$ & -2.18 & 0.46 & -4.71 & $<0.001$ \\
\hline FM & 8.4 & 3.16 & 2.66 & 0.009 & 0.6 & 0.19 & 3.12 & 0.002 \\
\hline Tamb & -5.16 & 2.82 & -1.83 & 0.069 & -0.29 & 0.17 & -1.69 & 0.093 \\
\hline
\end{tabular}

Associations were assessed by a univariate analysis of variance approach. BMI, body mass index; CE, cold exposure; FM, fat mass; FFM, fat free mass; REE, resting energy expenditure; Std Error, standard error; Tamb, ambient temperature; Texp, exposure temperature; TN, thermoneutrality; $\triangle \mathrm{REE}$, change of REE after $\mathrm{CE}$.

\section{Changes in REE}

Basal REE was significantly higher in the OB group compared to the NW group $(p<0.001)$. This difference remained statistically significant after adjustment for FFM and age ( $p<0.001$ data not shown). There was no statistical difference in REE at TN between the OW and
OB groups ( $p=0.079$, data not shown). In the whole cohort, REE increased from baseline 1,619 [IQR: 1,438$1,818]$ to $1,700[1,503-1,934] \mathrm{kcal} /$ day $(p<0.001)$ after $\mathrm{CE}$ with a high interindividual variance. While we observed an overall increase of $6.5 \%$ following $\mathrm{CE}(p<0.001)$ in the NW group, there was no significant change in the 


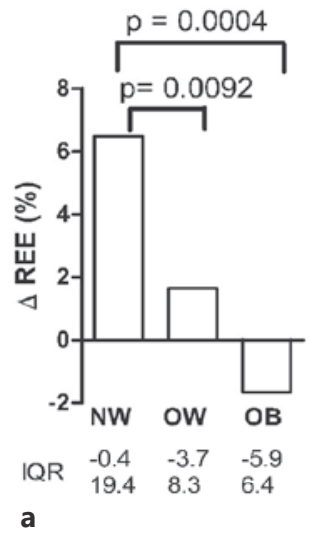

a

Fig. 1. a, b Changes in REE and RER after CE; for all participants and stratified for BMI group. Values are presented as median with IQR; Differences between BMI groups were assessed by the Kruskal-Wallis test; IQR, interquartile range; $\mathrm{NW}$, normal weight (BMI $18.5-24.9 \mathrm{~kg} / \mathrm{m}^{2}$ ); OW, overweight (BMI $25.0-29.9 \mathrm{~kg} / \mathrm{m}^{2}$ ); OB, obese (BMI $\left.\geq 30.0 \mathrm{~kg} / \mathrm{m}^{2}\right)$; $\Delta \mathrm{REE}$, differences in resting energy expenditure before and after $C E ; \triangle R E R$, differences in respiratory exchange ratio before and after $\mathrm{CE}$.

OW $(1.6 \%, p=0.468)$ or OB groups $(-1.7 \%, p=0.917$, Fig. 1), respectively. The change in REE was significantly different between BMI groups $(p<0.001)$. As shown in Table 2, this finding remained significant after adjustment for age, Texp, sex, and FFM in a multiple regression analysis $(p=0.019)$ which demonstrates a negative association between $B M I$ and $\triangle$ REE. Other putative predictors did not reveal an association with $\triangle \mathrm{REE}$ (online suppl. Table S2). While there was no significant association between fat ratio (FM [\%]) and $\triangle \operatorname{REE~}(\%)(p=0.245)$, we could observe an association after adjustment for age and gender $(p=0.036)$. There was a significant decrease in the respiratory exchange ratio after CE in all BMI groups with a tendency to decrease less depending on the BMI category, but there was no significant difference between groups (all $p>0.094$; Fig. 1).

\section{Temperature Monitoring}

The median outdoor temperature on study days was $5.1^{\circ} \mathrm{C}[1.3-10.1]$, which was comparable between BMI groups $(p=0.444)$. Room temperature was $22.0^{\circ} \mathrm{C}[21.0$ $22.0]$ at $\mathrm{TN}$ and $23.0^{\circ} \mathrm{C}$ [21.0-24.0] at CE. Both temperatures were comparable between BMI groups $(p=0.619$ and $p=0.356$, respectively). Participants started shivering at $19.9^{\circ} \mathrm{C}[18.1-21.6]$ with no significant difference be-

Thermogenic Response to Cold in

Relation to BMI tween BMI groups $(p=0.398)$. Likewise, Texp was similar in all BMI groups $\left(22.8^{\circ} \mathrm{C}\right.$ [21.0-24.3], $\left.p=0.106\right)$. There were significant differences in the water inlet temperature at the beginning of the experiment due to the following reason: while $32^{\circ} \mathrm{C}$ was the official starting temperature, some participants perceived this level as too warm. To avoid sweating and thus elevated REE, we decreased the temperature to achieve the thermoneutral zone of these participants. This temperature was significantly lower in the OB group $\left(31.0^{\circ} \mathrm{C}[30.1-32.0]\right)$ compared to the NW $\left(32.0^{\circ} \mathrm{C}[32.0-32.0], p<0.001\right)$ and $\mathrm{OW}\left(32.0^{\circ} \mathrm{C}[32.0-\right.$ $32.0], p=0.005)$ groups.

CE led to a significant decrease in overall skin temperature by $-3.5^{\circ} \mathrm{C}[-4.6$ to -2.8$](p<0.001)$. This decrease was not significantly different between BMI groups ( $p=0.891$; online suppl. Table S3). However, at TN and after CE, skin temperature was significantly higher in the NW group compared to the other two BMI groups ( $p<$ 0.001 for both conditions). Regarding skin temperature at the supraclavicular area (Tsupra), no changes in temperature were observed after CE (all $p>0.136$ ). However, Tsupra was significantly lower in the OB group compared to the NW and OW groups at TN and after CE (all $p<$ $0.001)$. In addition, there was a significant decrease in Tsupra in males from the NW and OB groups $(p=0.034$ and $p=0.002$, respectively). Linear regression showed that $\Delta$ Tsupra was not associated with BMI $(p=0.727)$.

\section{Cardiovascular Effects}

Systolic blood pressure (BP) significantly increased in the NW and OB groups $(p<0.001$ and $p=0.024$, respectively) during $\mathrm{CE}$, while the increase in systolic $\mathrm{BP}$ was not significant in the OW group $(p=0.235$; Table 3$)$. Diastolic BP significantly increased in all groups (all $p<$ 0.001). However, the magnitude of these increases was comparable between the BMI groups. The heart rate significantly decreased in all groups (all $p<0.002)$ and there were no significant differences between the BMI groups $(p=0.380)$.

\section{Circulating Metabolites}

We observed that fasting glucose levels decreased during CE in all BMI groups (all $p<0.002$ ). Neither basal values nor those after $\mathrm{CE}$ were significantly different between groups ( $p=0.172$ at TN and $p=0.876$ after CE). Furthermore, the relative decrease was comparable across groups ( $p=0.120$, Fig. 2a). Plasma insulin levels were higher in the $\mathrm{OB}$ group compared to the NW and OW groups at both conditions (both $p<0.001$ ). While CE had no significant effect on plasma insulin levels in the NW 


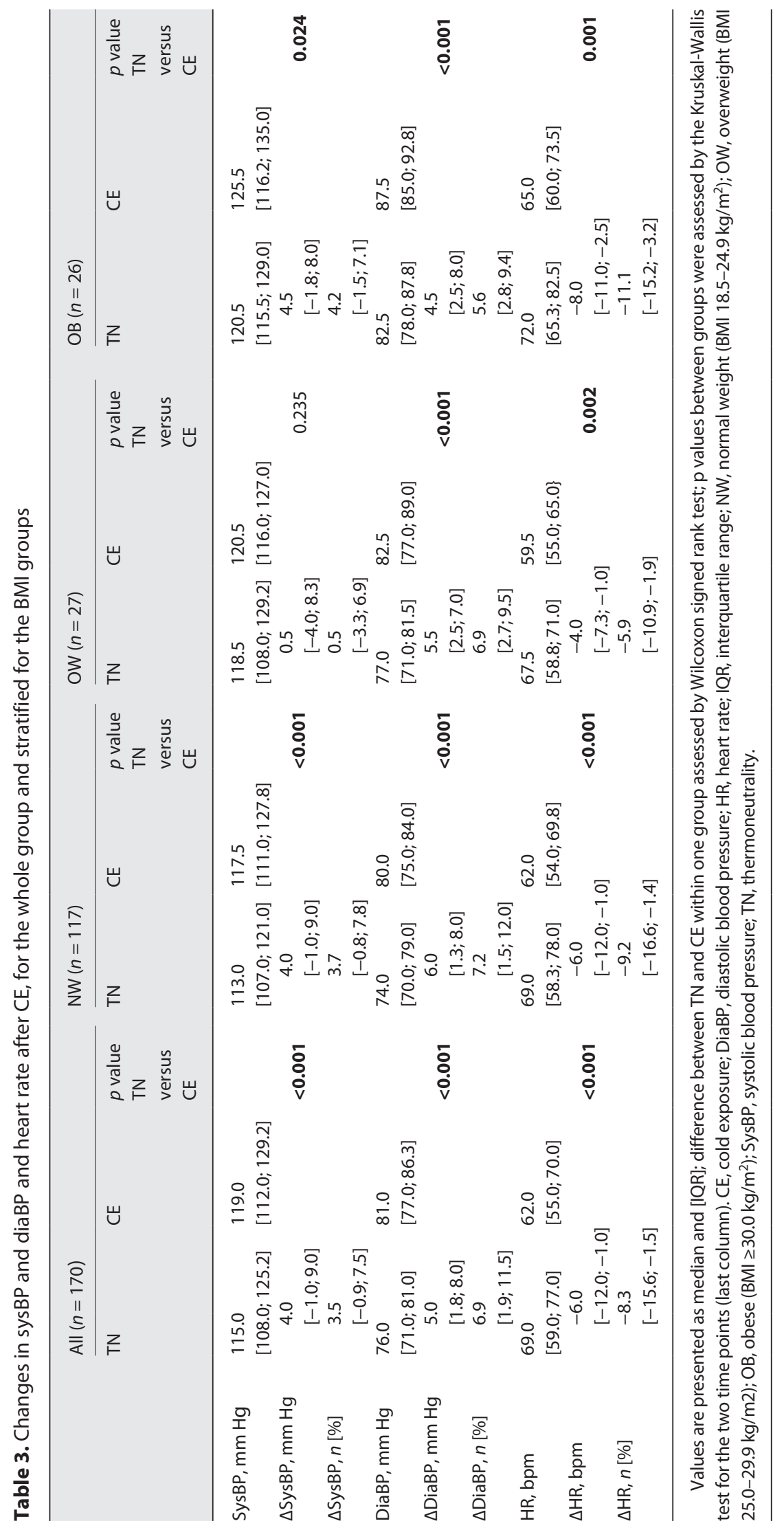


Fig. 2. a-g Changes in circulating metabolic and hormonal parameters upon CE; Data are presented as median with IQR; ${ }^{* * *} p<0.001 ;{ }^{* *} p<0.01 ;{ }^{*} p<0.05$ as assessed by Kruskal-Wallis analysis of variance with Bonferroni correction. HOMAIR, homeostasis model assessment of insulin resistance; NEFA, nonesterified fatty acids; NW, participants with BMI 18.5$24.9 \mathrm{~kg} / \mathrm{m}^{2}$ ( $\left.n=115\right)$; OW, participants with BMI $25-29.9 \mathrm{~kg} / \mathrm{m}^{2}(n=27)$; OB, participants with $\mathrm{BMI} \geq 30 \mathrm{~kg} / \mathrm{m}^{2}(n=26)$; TGs, triglycerides; $\Delta=\mathrm{CE}$ values - basal values.

Fig. 3. Expression of beiging markers in human scWAT: mRNA expression levels of the beiging markers UCP1, PGC1a, PRDM16, and CIDEA, measured by qPCR in human scWAT of 17 participants with elevated BMI ( 5 males and 12 females, BMI $28.0-41.2 \mathrm{~kg} / \mathrm{m}^{2}$ ). Importin 8 , peptidylprolyl isomerase $\mathrm{A}$, and glyceraldehyde 3-phosphate dehydrogenase mRNA levels served as HKGs for normalization of the data. Values are presented as fold change; ${ }^{*} p<0.05$ as assessed by Wilcoxon signed rank test. HKGs, housekeeping genes.
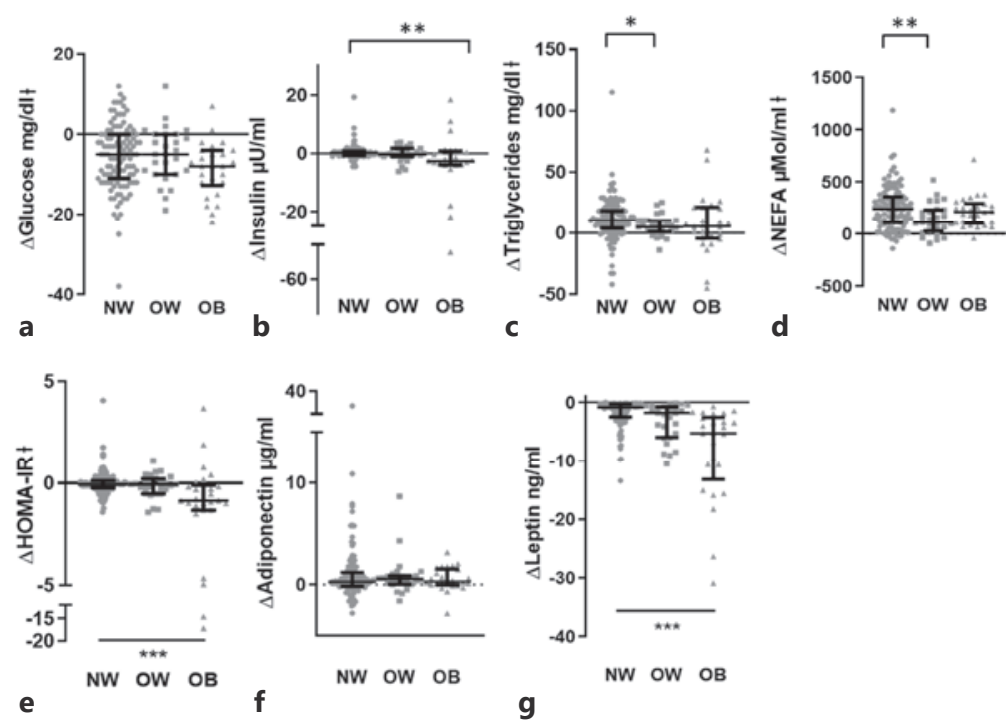

e

g

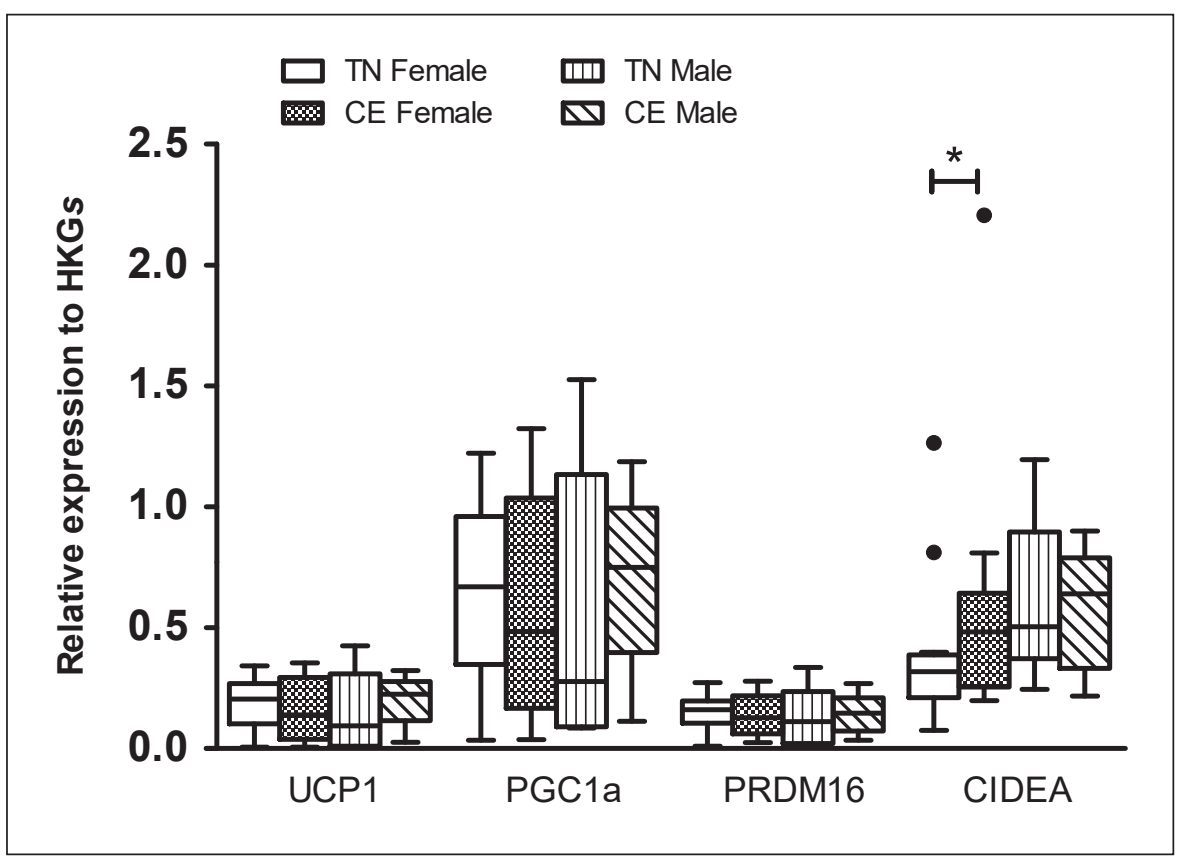

and OW groups, insulin levels decreased in the $\mathrm{OB}$ group by $2.6 \mu \mathrm{U} / \mathrm{mL}$ [ -44.1 to $-7.6 \%]$ ( $p=0.031$, Fig. $2 \mathrm{~b})$. At baseline, HOMA-IR as a crude measure of insulin resistance calculated from fasting glucose and insulin [23] was significantly higher in the OB group (3.40 [2.17-7.67]) compared to the OW (1.48 [0.66-2.12], $p<0.001)$ and NW groups (1.26 [0.71-1.96], each $p<0.001)$. After CE, HOMA-IR decreased significantly in the $\mathrm{OB}$ group by

Thermogenic Response to Cold in Relation to BMI
$0.87[-1.27$ to -0.17$]$ ( $p=0.003)$, whereas the levels in the NW and OW groups were unchanged $(p=0.067$ and $p=$ 0.248 , respectively, Fig. 2e).

We further observed that serum TG significantly increased during CE in the NW $(p<0.001)$ and OW $(p=$ $0.001)$ groups, but not in the OB group ( $p=0.084$, Fig. $2 c)$. Although TG levels at TN and after CE were lower in the NW and OW groups than in the OB group (both $p<$ 
$0.001)$, the increase in TG levels was significantly lower in the OB group $(p=0.041)$. Likewise, there was a significant increase in plasma NEFA in all groups (all $p<0.001$ ). This increase was significantly higher in the NW and $\mathrm{OB}$ groups compared to the OW group ( $p=0.024$, Fig. $2 \mathrm{~d}$ ). However, in males there was also a significant difference between the NW and $\mathrm{OB}$ groups with men in the NW group having a higher increase compared to those with obesity $(p=0.020)$.

Serum levels of adiponectin significantly increased during $\mathrm{CE}$ in all $\mathrm{BMI}$ groups (NW $p<0.001$; OW $p=$ 0.004 ; $\mathrm{OB} p=0.010)$, and the increase was comparable across BMI groups ( $p=0.856$, Fig. $2 \mathrm{f}$ ). In contrast, there was a significant decrease in plasma leptin concentrations after CE in all groups (all $p<0.001$ ). This decrease was highest in the $\mathrm{OB}$ group compared to the other groups $(p<0.001$, Fig. $2 \mathrm{~g})$. However, considering the relative decrease in leptin levels, no difference was observed between the BMI groups $(p=0.552)$.

Furthermore, we observed a significant increase in serum CRP levels in all BMI groups (all $p<0.014$ ), but this increase was comparable across BMI groups $(p=0.280)$. Basal levels and levels of CRP after CE were significantly higher in the OB group compared to the NW and OW groups ( $p<0.001$ each, data not shown).

\section{Relationship between Dietary Intake and \\ Cold-Induced Increase in REE}

As dietary habits may influence the response to mild $\mathrm{CE}$, we related total energy intake as well as macronutrient composition over 3 days prior to $\mathrm{CE}$ to cold-induced changes in REE. It turned out that there was no significant association of $\triangle \mathrm{REE}$ and $\triangle \mathrm{REE}(\%)$ with calorie intake ( $p$ $=0.511, p=0.329)$, nor percent fat intake $(p=0.456, p=$ $0.323)$, nor percent carbohydrate intake $(p=0.914, p=$ $0.970)$, nor percent protein intake $(p=0.061, p=0.051)$.

\section{mRNA Expression of scWAT}

To evaluate the impact of CE on beiging in WAT, we performed a marker gene expression analysis by qPCR in an exploratory approach. Beiging marker expression levels in paired scWAT samples from 17 obese participants revealed no significant changes between baseline and after CE for UCP1, PRDM16, and PGC1 $\alpha$ (all $p>$ 0.962), as demonstrated in Figure 3. However, there was a significant increase in cell death activator (CIDEA) mRNA in females $(p=0.034)$, whereas no significant change was seen in the much smaller subgroup of males $(p=0.313)$.

\section{Discussion}

This study addressed differences in the thermogenic and metabolic response to short-term mild CE across three BMI classes. We observed significant differences in changes of REE upon a 2-h CE between the BMI groups. While participants with normal weight showed a significant increase in REE by 6.5\%, participants with overweight or obesity did not exhibit a significant increase in $\mathrm{REE}$ after CE. Linear regression analysis revealed an inverse association between $B M I$ and $\triangle$ REE.

To date, reports on this topic from other research groups were conflicting $[5,17]$. These studies were carried out under less strict conditions and with much smaller sample sizes. In contrast, our large cohort of 173 adult participants undergoing a highly standardized and individualized cooling protocol after an overnight fast has a high potential to elucidate the relationship between BMI and changes in REE upon CE. However, due to the crosssectional design of the study, it remains unclear if the impaired thermogenic response to cold is a cause or consequence of obesity.

It is noteworthy that there was high interindividual variability in the thermogenic response to $\mathrm{CE}$ across the three BMI categories and it is likewise unclear which factors are driving this variability. Additionally, there was also a high variability in the metabolic changes among individuals of all BMI groups. This phenomenon is usually poorly recognized but may point to a general high interindividual variability in metabolic functions. Such variability is also observed after defined challenges as reported by our group in a previous study performed on healthy men [25].

It is interesting to note that TGs increased to a higher magnitude in participants with normal weight compared to the OW and OB groups. An overall increase in TGs and NEFAs upon CE was also described by others [26-28]. It is known that BAT not only preferentially oxidizes intracellular fatty acids but can also metabolize other substrates such as glucose [29]. This may explain why we saw a decrease in plasma glucose concentrations in all groups, in agreement with previous observations on the effect of CE on glucose uptake into BAT $[30,31]$.

Insulin levels decreased significantly in the OB group, but not in the NW and OW groups after CE. Likewise, HOMA-IR significantly improved supporting the concept that CE might be beneficial for insulin sensitivity and whole-body glucose homeostasis, particularly in insulinresistant persons $[14,30]$. Along this line, we recently showed that mild CE induces Tregs in WAT, suggesting 
a local immunosuppressive and anti-inflammatory action [32].

Skin temperatures were slightly lower in participants with overweight and obesity at TN and after CE compared to participants with normal weight. Despite this difference, the decrease in skin temperature was comparable across groups which is in accordance with the published literature $[5,33]$. A possible explanation is that a higher body FM may lead to better insulation, resulting in lower skin temperature of participants with obesity [34]. This improved insulation may be beneficial for maintaining a stable core temperature and may shift the thermoneutral zone to lower skin temperatures [35]. This phenomenon was also observed in our study. Thus, individuals with obesity may not need to activate their metabolism in order to defend core temperature as readily as persons with normal weight [36]. However, cold perception was similar across BMI categories in our study which resulted in comparable shivering and, thus, exposure temperatures. This indicates that individuals with normal weight increase their REE at mild cold mainly via NST and subjects with a higher BMI have impaired NST leading to a similar shivering threshold even though their thermoneutral zone is at lower temperatures. Tsupra, measured over the location with a residual BAT depot, was significantly lower in the OB group which may also be explained by lower supraclavicular BAT activity. Superficial skin temperature in the supraclavicular area was reported to be associated with BAT activity in women [37].

We were also interested to investigate whether CE may lead to changes in the expression of browning markers in biopsies from scWAT. However, this could only be done in a subgroup of volunteers with overweight and obesity. Among the selected markers, CIDEA mRNA levels increased after $\mathrm{CE}$ in both male and female participants. However, this upregulation reached statistical significance only in the female participants. CIDEA codes for a lipid droplet-associated protein, which has been reported to correlate with insulin sensitivity and lipid disorders in persons with obesity [38]. CIDEA protein has also been shown to regulate UCP1 expression in human adipocytes and as a result is known to coregulate browning, although there was no significant change in UCP1 expression upon CE. A possible explanation is that the short-term duration of CE may have not allowed to elicit significant changes in UCP1 mRNA expression.

In our study, samples of scWAT were taken immediately after each REE measurement. In a recent study, fat biopsies were collected $4 \mathrm{~h}$ after CE [39]. In the same study,
scWAT samples were taken in summer and winter, but there was an increase in UCP1 and PGC1 $\alpha$ expression after $\mathrm{CE}$ only in the samples collected during summer. The authors postulated that mRNA expression of these markers is already high in winter due to natural CE and that additional CE does not lead to a further increase. Additionally, they saw no effect of CE in participants with a BMI of over 30 $\mathrm{kg} / \mathrm{m}^{2}$, regardless of season [39]. As our biosamples were collected only during the winter months and only from individuals with obesity, this could explain why we were unable to see changes in the expression of those genes. In contrast to another group, we could not detect higher basal levels of UCP1 mRNA expression in females compared to males [40]. However, despite having comparable basal values of CIDEA mRNA expression in men and women, which is in line with another study [41], the observed increase in CIDEA mRNA expression in women upon CE may also indicate a sex-specific difference in browning of scWAT. This finding is consistent with previous reports suggesting that females have a higher browning capacity [42, 43]. However, our recent analysis of potential sex differences in thermogenic response to cold did not provide sufficient evidence for a sex difference [20].

\section{Strengths and Limitations}

The study population was rather young and healthy and was of Caucasian origin eliminating age, ethnicity, and disease as potential confounders. Therefore, the results might not be valid for the general population and specific population groups such as other ethnicities or elderly. Furthermore, the subgroup of participants with obesity consisted largely of individuals with grade 1 obesity, so we cannot draw conclusions about individuals with more severe obesity.

The increase in REE upon CE as described here may be largely due to an increase in NST, but we cannot exclude shivering of skeletal muscle contributing to this increase in energy expenditure. Therefore, an individualized cooling protocol was applied to assess the onset of shivering. Participants were also asked to report any signs of involuntary muscle contractions. By increasing the temperature at the slightest sign of shivering, we are convinced that muscle shivering was minimized. We did not apply surface electromyography, as only surface muscle shivering can be ruled out by this method, but not shivering of deep muscles. Participants who showed signs of shivering during the second REE measurement were excluded from the analysis. Furthermore, we did not directly measure BAT activity, therefore, our results might not reflect true cold-induced BAT activation. 
The capacity for NST is subject to intraindividual variation [33]. Participants in our setting were only exposed to cold on a single study day. During the establishment of the protocol, we exposed 3 volunteers 3 times to the same $\mathrm{CE}$ and found a coefficient variation under $10 \%$ (data not shown). Together with the rigorous application of the protocol and the large number of study participants, we are rather confident that the decrease in NST upon CE with increasing BMI is rather robust. However, there is a possibility that we could not utilize the full capacity of cold-induced thermogenesis under fasting conditions and we cannot exclude a different picture under feeding.

Due to the limited amount of biopsy material we had to restrict our analysis of changes in gene expression. Further studies should address the expression of other genes that are important for non-UCP futile cycling or insulin signaling.

In conclusion, cold-induced thermogenesis, assessed by the increase in REE upon defined CE, was found to be impaired in individuals with overweight and obesity. If this impairment is caused by a higher FM or if a higher BMI is due to impaired thermogenesis cannot be answered due to the cross-sectional design of the study. The results also suggest that $\mathrm{CE}$ improves insulin sensitivity in participants with obesity underpinning the beneficial effects of CE on metabolism. Further research is warranted to explore the long-term effects of cold-induced thermogenesis on body weight and metabolism.

\section{Acknowledgments}

We are grateful for the statistical advice provided by Lynne Stecher, Jakob Linseisen, and Nina Grundmann. We thank Karin Lietzau, Simone Herkenhoff, and Irmgard Sperrer for technical assistance. Furthermore, we thank Theresa Britzl for technical support at the Munich study center. Finally, we thank all study participants.

\section{Statement of Ethics}

This study protocol was reviewed and approved the Ethics Commission of the School of Medicine of the Technical University of Munich (\#236/16S).

Each volunteer gave their written informed consent.

\section{Conflict of Interest Statement}

The authors have no conflicts of interest to declare.

\section{Funding Sources}

This work was supported by the ZIEL Institute for Food and Health, Freising, and the Else Kroener-Fresenius Foundation, Bad Homburg, Germany. The funding body was not involved in the study design, data collection and analysis, decision to publish, or manuscript preparation.

\section{Author Contributions}

Hans Hauner and Melina Claussnitzer conceived and designed the study. Laura Aline Mengel, Bahareh Nemati Moud, Hatti Seidl, Alberto Mesas-Fernández, and Claudine Seeliger performed the experiments. Laura Aline Mengel analyzed data and prepared the manuscript. Christina Holzapfel, Beate Brandl, and Thomas Skurk assisted with experimental design, recruitment of volunteers, and data interpretation. All authors were involved in the final revision of the manuscript.

\section{Data Availability Statement}

All data generated or analyzed during this study are included in this article and its online supplementary Files. Further enquiries can be directed to the corresponding author.

\section{References}

1 Hatai S. On the presence in human embryos of an interscapular gland corresponding to the so-called hibernating gland of lower mammals. Anat Anz. 1902;21:369-73.

2 Bonnot E. The interscapular gland. J Anat Physiol. 1908 Oct;43(Pt 1):43-58.

3 Rodgers RJ, Tschöp MH, Wilding JP. Antiobesity drugs: past, present and future. Dis Model Mech. 2012 Sep;5(5):621-6.

4 Virtanen KA, Lidell ME, Orava J, Heglind M, Westergren R, Niemi $T$, et al. Functional brown adipose tissue in healthy adults. $\mathrm{N}$ Engl J Med. 2009 Apr;360(15):1518-25.
5 van Marken Lichtenbelt WD, Vanhommerig JW, Smulders NM, Drossaerts JM, Kemerink GJ, Bouvy ND, et al. Cold-activated brown adipose tissue in healthy men. $\mathrm{N}$ Engl J Med. 2009 Apr;360(15):1500-8.

6 Cypess AM, Lehman S, Williams G, Tal I, Rodman D, Goldfine AB, et al. Identification and importance of brown adipose tissue in adult humans. N Engl J Med. 2009 Apr; 360(15):1509-17.

7 Brendle C, Werner MK, Schmadl M, la Fougère C, Nikolaou K, Stefan N, et al. Correlation of brown adipose tissue with other body fat compartments and patient characteristics. Acad Radiol. 2018 Jan;25(1):102-10.
8 Dadson P, Hannukainen JC, Din MU, Lahesmaa M, Kalliokoski KK, Iozzo P, et al. Brown adipose tissue lipid metabolism in morbid obesity: effect of bariatric surgery-induced weight loss. Diabetes Obes Metab. 2018 May;20(5):1280-8.

9 Cannon B, Nedergaard J. Brown adipose tissue: function and physiological significance. Physiol Rev. 2004;84(1):277-359.

10 Ouellet V, Routhier-Labadie A, Bellemare W, Lakhal-Chaieb L, Turcotte E, Carpentier AC, et al. Outdoor temperature, age, sex, body mass index, and diabetic status determine the prevalence, mass, and glucose-uptake activity of 18 F-FDG-detected BAT in humans. J Clin Endocrinol Metab. 2011 Jan;96(1):192-9. 
11 Leitner BP, Huang S, Brychta RJ, Duckworth CJ, Baskin AS, McGehee S, et al. Mapping of human brown adipose tissue in lean and obese young men. Proc Natl Acad Sci. 2017 Aug;114(32):8649-54.

12 Yoneshiro T, Aita S, Matsushita M, Okamatsu-Ogura Y, Kameya T, Kawai Y, et al. Agerelated decrease in cold-activated brown adipose tissue and accumulation of body fat in healthy humans. Obesity. 2011 Sep;19(9): 1755-60.

13 Franz D, Weidlich D, Freitag F, Holzapfel C, Drabsch T, Baum T, et al. Association of proton density fat fraction in adipose tissue with imaging-based and anthropometric obesity markers in adults. Int J Obes. 2018 Feb;42(2): 175-82.

14 Chondronikola M, Volpi E, Borsheim E, Porter C, Annamalai P, Enerback S, et al. Brown adipose tissue improves whole-body glucose homeostasis and insulin sensitivity in humans. Diabetes. 2014 Dec;63(12):4089-99.

15 Matsushita M, Yoneshiro T, Aita S, Kameya $\mathrm{T}$, Sugie H, Saito M. Impact of brown adipose tissue on body fatness and glucose metabolism in healthy humans. Int J Obes. 2014 Jun; 38(6):812-7.

16 Hanssen MJW, Wierts R, Hoeks J, Gemmink A, Brans B, Mottaghy FM, et al. Glucose uptake in human brown adipose tissue is impaired upon fasting-induced insulin resistance. Diabetologia. 2015 Mar;58(3):586-95.

17 Hanssen MJW, van der Lans AAJJ, Brans B, Hoeks J, Jardon KMC, Schaart G, et al. Shortterm cold acclimation recruits brown adipose tissue in obese humans. Diabetes. 2016 May; 65(5):1179-89.

18 Wibmer AG, Becher T, Eljalby M, Vaughan R, Schö H, Cohen P, et al. Brown adipose tissue is associated with healthier body fat distribution and metabolic benefits independent of regional adiposity. Cell Reports Med. 2021;2: 100332.

19 Becher T, Palanisamy S, Kramer DJ, Eljalby M, Marx SJ, Wibmer AG, et al. Brown adipose tissue is associated with cardiometabolic health. Nat Med. 2021;27(1):58-65.

20 Mengel LA, Seidl H, Brandl B, Skurk T, Holzapfel C, Stecher L, et al. Gender differences in the response to short-term cold exposure in young adults. J Clin Endocrinol Metab. 2020 May;105(5):e1938-48.

21 ISO. ISO9886. Evaluation of thermal strain by physiological measurements. Geneva: International Standards Organization; 2004.

22 Weir JB. New methods for calculating metabolic rate with special reference to protein metabolism. J Physiol. 1949 Aug;109(1-2): $1-9$.
23 Fullmer S, Benson-Davies S, Earthman CP, Frankenfield DC, Gradwell E, Lee PSP, et al. Evidence analysis library review of best practices for performing indirect calorimetry in healthy and non-critically ill individuals. J Acad Nutr Diet. 2015 Sep;115(9):1417-46.e2.

24 Matthews DR, Hosker JP, Rudenski AS, Naylor BA, Treacher DF, Turner RC. Homeostasis model assessment: insulin resistance and beta-cell function from fasting plasma glucose and insulin concentrations in man. Diabetologia. $1985 \mathrm{Jul} ; 28(7): 412-9$.

25 Krug S, Kastenmüller G, Stückler F, Rist MJ, Skurk T, Sailer M, et al. The dynamic range of the human metabolome revealed by challenges. FASEB J. 2012 Jun;26(6):2607-19.

26 Weir G, Ramage LE, Akyol M, Rhodes JK, Kyle CJ, Fletcher AM, et al. Substantial metabolic activity of human brown adipose tissue during warm conditions and cold-induced lipolysis of local triglycerides. Cell Metab. 2018 Jun;27(6):1348-55.e4.

27 Blondin DP, Labbé SM, Tingelstad HC, Noll C, Kunach M, Phoenix S, et al. Increased brown adipose tissue oxidative capacity in cold-acclimated humans. J Clin Endocrinol Metab. 2014 Mar;99(3):E438-46.

28 Vosselman MJ, van der Lans AAJJ, Brans B, Wierts R, van Baak MA, Schrauwen P, et al. Systemic-adrenergic stimulation of thermogenesis is not accompanied by brown adipose tissue activity in humans. Diabetes. 2012 Dec; 61(12):3106-13.

29 Blondin DP, Frisch F, Phoenix S, Guérin B, Turcotte ÉE, Haman F, et al. Inhibition of intracellular triglyceride lipolysis suppresses cold-induced brown adipose tissue metabolism and increases shivering in humans. Cell Metab. 2017 Feb;25(2):438-47.

30 Iwen KA, Backhaus J, Cassens M, Waltl M, Hedesan OC, Merkel M, et al. Cold-induced brown adipose tissue activity alters plasma fatty acids and improves glucose metabolism in men. J Clin Endocrinol Metab. 2017 Nov; 102(11):4226-34.

31 Leitner BP, Weiner LS, Desir M, Kahn PA, Selen DJ, Tsang C, et al. Kinetics of human brown adipose tissue activation and deactivation. Int J Obes. 2019 Mar;43(3):633-7.

32 Becker M, Serr I, Salb VK, Ott VB, Mengel L, Blüher $M$, et al. Short-term cold exposure supports human Treg induction in vivo. Mol Metab. 2019 Oct;28:73-82.

33 Brychta RJ, Huang S, Wang J, Leitner BP, Hattenbach JD, Bell SL, et al. Quantification of the capacity for cold-induced thermogenesis in young men with and without obesity. J Clin Endocrinol Metab. 2019 Oct;104(10):486578.
34 Castellani JW, Young AJ. Human physiological responses to cold exposure: acute responses and acclimatization to prolonged exposure. Auton Neurosci. 2016 Apr;196:63-74.

35 Moellering DR, Smith DL. Ambient temperature and obesity. Curr Obes Rep. 2012 Mar; 1(1):26-34.

36 Speakman JR. Obesity and thermoregulation. Handbook of clinical neurology. Elsevier; 2018. p. 431-43.

37 Martinez-Tellez B, Garcia-Rivero Y, SanchezDelgado G, Xu H, Amaro-Gahete FJ, Acosta FM, et al. Supraclavicular skin temperature measured by iButtons and 18F-fluorodeoxyglucose uptake by brown adipose tissue in adults. J Therm Biol. 2019 May;82:178-85.

38 Montastier E, Déjean S, Le Gall C, Saris WH, Langin D, Viguerie N. Adipose tissue CIDEA is associated, independently of weight variation, to change in insulin resistance during a longitudinal weight control dietary program in obese individuals. PLoS One. 2014 Jul;9(7): e98707.

39 Kern PA, Finlin BS, Zhu B, Rasouli N, McGehee RE, Westgate PM, et al. The effects of temperature and seasons on subcutaneous white adipose tissue in humans: evidence for thermogenic gene induction. J Clin Endocrinol Metab. 2014 Dec;99(12):E2772-9.

40 Barquissau V, Léger B, Beuzelin D, Martins F, Amri E-Z, Pisani DF, et al. Caloric restriction and diet-induced weight loss do not induce browning of human subcutaneous white adipose tissue in women and men with obesity. Cell Rep. 2018 Jan;22(4):1079-89.

41 Gummesson A, Jernås M, Svensson PA, Larsson I, Glad CAM, Schéle E, et al. Relations of adipose tissue CIDEA gene expression to basal metabolic rate, energy restriction, and obesity: population-based and dietary intervention studies. J Clin Endocrinol Metab. 2007; 92(12):4759-65.

42 Scalzo RL, Peltonen GL, Giordano GR, Binns SE, Klochak AL, Paris HLR, et al. Regulators of human white adipose browning: evidence for sympathetic control and sexual dimorphic responses to sprint interval training. PLoS One. 2014 Mar;9(3):e90696.

43 Otero-Díaz B, Rodríguez-Flores M, SánchezMuñoz V, Monraz-Preciado F, OrdoñezOrtega S, Becerril-Elias V, et al. Exercise induces white adipose tissue browning across the weight spectrum in humans. Front Physiol. 2018 Dec;9:1781.
Thermogenic Response to Cold in Relation to BMI
Obes Facts 2022;15:405-415 